\title{
Is the concept of clinical equipoise still relevant to research?
}

This article (BMJ 2017;359:j5787, doi:10.1136/bmj.j5787) should have acknowledged the Laura and John Arnold

Foundation for its support of the work of Spencer Hey and for funding a conference on equipoise and innovative trial designs, where the article's ideas and arguments were discussed. 